OPEN ACCESS

Edited by:

Renee Heffron,

University of Washington,

United States

Reviewed by:

Dvora Joseph Davey,

University of California, Los Angeles,

United States

*Correspondence:

Bethany Young Holt

byh@cami-health.org

Specialty section: This article was submitted to HIV and STIS,

a section of the journal Frontiers in Reproductive Health

Received: 04 May 2021

Accepted: 12 August 2021

Published: 06 September 2021

Citation:

Young Holt B, Turpin JA and Romano J (2021) Multipurpose

Prevention Technologies: Opportunities and Challenges to Ensure Advancement of the Most Promising MPTs.

Front. Reprod. Health 3:704841. doi: 10.3389/frph.2021.704841

\section{Multipurpose Prevention Technologies: Opportunities and Challenges to Ensure Advancement of the Most Promising MPTs}

\author{
Bethany Young Holt ${ }^{1 *}$, Jim A. Turpin ${ }^{2}$ and Joseph Romano ${ }^{3}$ \\ ${ }^{1}$ Initiative for Multipurpose Prevention Technologies, CAMI Health, Public Health Institute, Sacramento, CA, United States, \\ ${ }^{2}$ Howpin Consulting, Frederick, MD, United States, ${ }^{3}$ NWJ Group, LLC, Wayne, PA, United States
}

Keywords: multipurpose prevention technologies, hiv prevention, STI prevention, contraception, MPTs, sexual and reproductive health

\section{INTRODUCTION}

The HIV/sexually transmitted infection (STI) syndemics and the unmet need for modern contraceptive methods continue to pose significant health risks for women worldwide (1). Women are at high risk of HIV in many regions of the world, particularly young women and girls in SubSaharan Africa where $71 \%$ of all new infections are among adolescents (2-4). The risk of HIV acquisition and transmission through mother-to-child transmission (MTCT) and among pregnant and breastfeeding women are significant contributors to the HIV epidemic (5). Rates of STIs, such as gonorrhea, chlamydia, syphilis, and herpes are rising and compounding the risk of HIV acquisition $(6,7)$. At the same time, an estimated 218 million women in lower- and middleincome countries (LMICs) have an unmet need for contraception (8), and 817 women die each day from preventable causes related to pregnancy or child-birth (7). As awareness of the need to address these interlinked risks has increased, the need for new technologies that combine protection against unintended pregnancy, HIV and other STIs is a growing research priority (9). Addressing these interlinked risks also aligns with the goals of the WHO-led initiative for the elimination of maternal-to-child transmission (EMTCT) of HIV and syphilis as a public health priority (5).

Multipurpose Prevention Technologies (MPTs) are products that simultaneously prevent HIV, other STIs, and/or unintended pregnancy. They have power to revolutionize women's health by providing prevention for multiple indications (10). Additionally, MPTs that combine HIV prevention and contraception may improve uptake of and adherence to HIV pre-exposure prophylaxis (PrEP) by offering streamlined product delivery and eliminating the need for multiple, separate clinic visits to address family planning and other sexual and reproductive health (SRH) needs (11). Given the urgent need to reduce HIV infection in pregnant and breastfeeding women, MPTs that allow for contraception and prevent HIV and other STIs may benefit women who wish to conceive as well as pregnancy and breast feeding women who are not using contraceptives. A study among pregnant women of the dapivirine ring and the Truvada oral tablet aims to assess the safety, adherence and acceptability of these HIV prevention approaches when used during pregnancy which can inform MPT development (12). Yet, even with the potential of MPTs to transform the lives of women everywhere, especially those in LMICs, MPT development is scientifically and logistically complex. The resources critical for transitioning promising pre-clinical product candidates and formulations into clinical evaluation remain limited despite intensified collaborations and investments between government and private sectors. 


\section{OPPORTUNITIES}

Male and female condoms, when used properly through their multi-spectrum ability to block infection and transmission of HIV and other STIs and prevent pregnancy, are the only currently approved MPT products. The United Nations (UN) Department of Economic and Social Affairs recently reported a worldwide increase in condom use from 1994 to 2015 (13). The global condom market was valued at $\$ 6.76$ billion in 2017 and is estimated to increase to $\$ 11.1$ billion by 2023 (14). Yet, for many people condoms are not feasible for a variety of reasons. These include coital dependency, unequal power balance between male and female partners and other challenges that may arise in negotiating condom use in intimate relationships, and sexual preferences that play into condom disuse. Despite these many challenges, alternative contraceptive use and acceptability have markedly increased since the creation of various modern methods [such as the pill and the intrauterine device (IUD)] (13). However, the most commonly used contraceptive methods, namely sterilization, the pill, injectables and IUDs, do not protect against STIs. Thus, condoms are only a precursor to other successful MPTs, in which various drug delivery platforms (e.g., pills, injectables, vaginal rings, and subdermal implants etc) can be leveraged as more user-friendly options.

Over the past decade there has been a growing array of new MPT candidates proposed, with over two dozen in active development. These include intravaginal rings, vaginal and rectal gels, vaginal inserts and films, systemic delivery implants, subdermal microarray patches, and oral tablets containing contraceptives, anti-HIV and/or other STI prevention drugs (15) (Figure 1). The majority of MPT candidates are in early preclinical stages of development by small biotechnology companies and academic labs. These efforts are largely funded by the United States government, primarily the United States Agency for International Development (USAID) and the National Institutes of Health (NIH).

Multiple end-user preference studies are lending support to the potential reproductive health impact of MPTs, as well as informing on product design strategies. For example, an end-user study conducted in three sub-Saharan Africa countries indicates specific end-user preferences identifying preferred MPT product dosage forms (16). The TRIO Study explored user preferences after use of placebo formulations of oral tablets, intravaginal rings and injectables (17). Further insight into the potential success of long acting MPTs can also be inferred from a study of preference for long-acting injectable PrEP conducted among African and US women (18). In the US, several studies have specifically gauged women's preferences for MPTs $(19,20)$, including a crosssectional national survey that assessed women's preferences for MPTs in the form of injectables, vaginal gels, intravaginal rings and diaphragms. Results from these studies indicate a high level of end-user preference for female controlled, discreet, long-acting products, such as injectables. These finding are harmonious with decades of research on contraceptive methods demonstrating use is greater when more method options are available (21). Providing women with an array of MPT method options must then be a goal of any long term MPT development efforts and is essential in creating an MPT product portfolio that realizes the reproductive health impact of MPTs.

Although a start has been made in creating a new generation of MPT products, significant funding is required to bring these MPTS to market. This includes funds to support preclinical research for translation to clinical testing, support of clinical testing and small- and large-scale manufacturing. Importantly, this also includes investments of funds, time and expertise to address the complex regulatory requirements that will enable multi-indication MTPs to advance to regulatory approval. Because of this complexity, public and private partnerships between academic researchers, small companies, big pharmaceuticals, the USG, and other supporting groups are needed to foster an end- to-end approach while promoting the advancement of economically viable end-user friendly MPT products. Although resources remain limited, support for MPTs is growing within the U.S. Health and Human Services (22) as well as among European funders and life science investors. Many funding agencies that support MPTs are working to leverage and optimize limited resources to address priorities and gaps to advance the most promising candidates; such strategic collaborations are essential.

The current MPT field is building upon lessons learned from decades of research in contraception, HIV topical microbicide development, systemic HIV prevention products and prevention of non-HIV STIs. A number of single indication contraceptive products are available to women (23-25) and new innovations are also underway for male contraception $(25,26)$. Single indication HIV-only prevention products are also in development. Importantly, after multiple clinical trials demonstrating efficacy, the dapivirine intravaginal ring for HIV prevention is in the process of gaining regulatory approval in individual countries (27-30). Furthermore, long-acting injectable cabotegravir (CAB-LA) has completed phase 3 trials in men who have sex with men, transgender women, and cisgender women, showing strong efficacy and safety results $(31,32)$. Likewise, a growing number of HIV prevention combination products are in development that include two oral PrEP compounds approved for use: Truvada ${ }^{\circledR}$ for use by men and women containing emtricitabine and tenofovir disoproxil fumarate $(33,34)$, and Descovy ${ }^{\circledR}$, containing emtricitabine and tenofovir alafenamide, which currently is only approved for men who have sex with men (MSM) and transgender women (35). New approaches for prevention of non-HIV STIs include those which address growing concerns around the development of antibiotic resistant STI prevention $(36,37)$. These include agents which stimulate host immune responses as well as non-immune approaches, including vaginal barrier methods, vaginal biofilm inhibitors, and microbiome modulators (38-42). As with contraceptives, all of these single indication anti-infective products and combination HIV prevention products can be critical components of a future MPT strategy since they can serve as the building blocks for multi-indication MPTs.

\section{CHALLENGES}

The increasing number of technology options and new drugs entering the prevention pipeline will require proper 


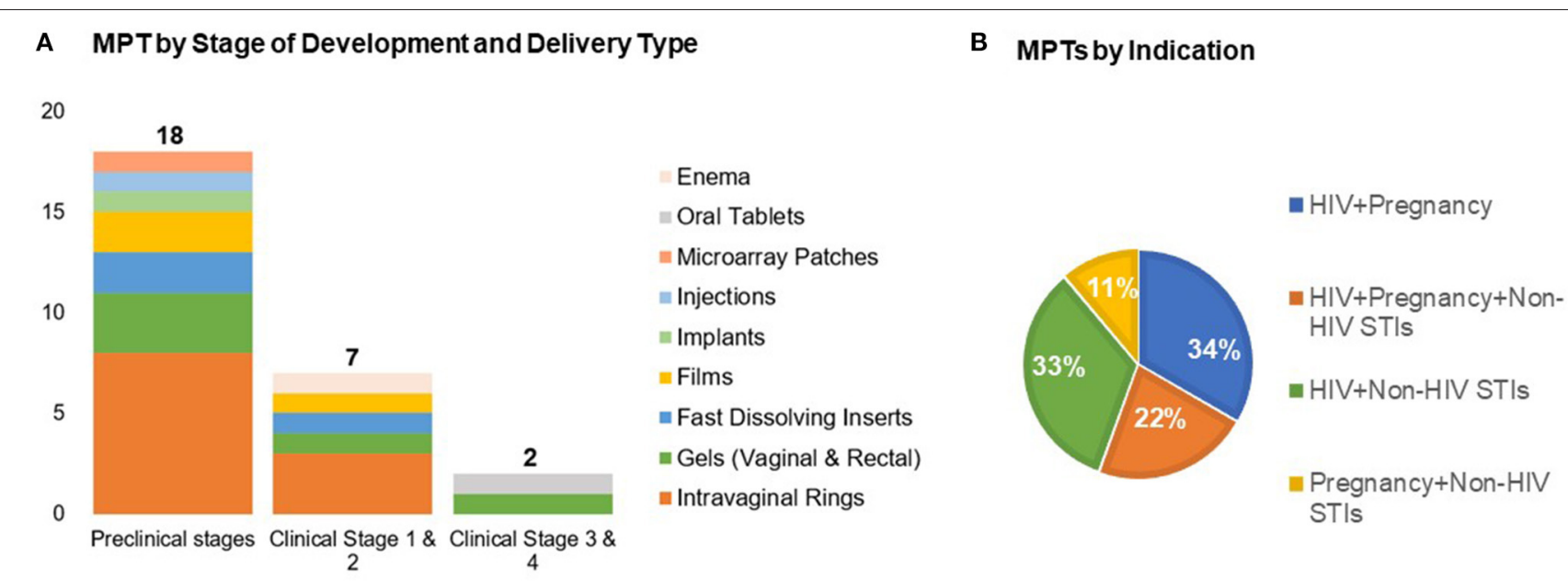

FIGURE 1 | Recent advances in MPTs development by (A) stage of development across delivery types and (B) indication $(n=27)$. Adapted from the Initiative for MPTs (IMPT) pipeline database (15).

investment to confirm that adequate resources are available to support potential high impact products through licensure and access needs. Challenges include obstacles to manufacturing (e.g., mixing of hormone and non-hormone drug substances/products), clinical trials (e.g., design of Phase 3 multi-indication trials) and other regulatory challenges (e.g., requirements for licensure of a combination dual indication long-acting product in a potentially novel drug-delivery system). Future trials will need to move beyond placebo-controlled designs toward superiority and non-inferiority trials that compare any new candidate against existing effective treatment options. As more single indication prevention products become available, the less likely it will be that regulatory bodies will approve efficacy studies that are placebo controlled. This approach will require larger more expensive trials with more challenging logistics, particularly if products like the CAB-LA HIV prevention injectable or EFDA implants become the standard of care (SOC) for HIV prevention (at least for LA systemic products) $(42,43)$. Further, although concurrent development of promising MPT candidates can accelerate progress for the field, available limited resources should be invested in a portfolio of diverse promising approaches for indication, mechanism of action and dosage form, and avoid developing nearly identical MPT products without strong justification for such an investment.

Promoting the development of a product pipeline that combines and optimizes the expertise currently associated with single indication products to create the desired multi-indication MPTs is key. This will require integration of the preclinical, clinical manufacturing and regulatory expertise associated with STI and contraceptive product development into a focused platform capable of supporting the development and licensure of multi-indication MPTs. This effort will require not only clarifying the complex manufacturing and regulatory challenges associated with combining multiple drugs and excipients, that may have incompatible biophysical, rheological and biochemical properties, but also the creation of multidisciplinary public/private partnerships to fund and guide this effort. Central, too, for the success of MPTs is the technical guidance required to evaluate and advance promising preclinical products into clinical formulations that can be advanced to human testing and ultimately licensure, particularly for groups with little experience in these areas $(44,45)$.

\section{DISCUSSION}

MPTs present significant reproductive health and general opportunities for addressing multiple indications in at-risk populations, particularly adolescent girls and young women in regions of the world where risk of HIV, other STIs and unintended pregnancies remains high. Given the current limited resources for expansion of MPT product development, ongoing strategic thinking and action is needed to optimize use of technical capacities, enhance collaborative approaches, identify resources to help fill gaps, and add rigor to the development process with the aim of advancing the most promising products.

\section{AUTHOR CONTRIBUTIONS}

All authors listed have made a substantial, direct and intellectual contribution to the work, and approved it for publication.

\section{FUNDING}

The authors declare that this work was made possible by the generous support of the Eunice Kennedy Shriver National Institute of Child Health and Human Development (NICHD) through an interagency agreement with the United States Agency for International Development (USAID), cooperative agreement number AID-OAA-A-16-00045. The funder had no involvement in this manuscript. The funder was not involved in the study design, collection, analysis, interpretation of data, and the writing of this article or the decision to submit it for publication. 


\section{ACKNOWLEDGMENTS}

The authors would like to thank Anke Hemmerling (University of California San Francisco \& University of California Berkeley),

\section{REFERENCES}

1. Deese J, Philip N, Lind M, Ahmed K, Batting J, Beksinska M, et al. Sexually transmitted infections among women randomized to depot medroxyprogesterone acetate, a copper intrauterine device or a levonorgestrel implant. Sex Transm Infect. (2020) 97:1-17. doi: 10.3389/frph.2021.668685

2. Joint United Nations Programme on HIV/AIDS (UNAIDS). How AIDS Changed Everything-MDG6:15 Years, 15 Lessons of Hope from the AIDS Response. Geneva: UNAIDS (2015).

3. Hosek S, Celum C, Wilson CM, Kapogiannis B, Delany-Moretlwe S, Bekker LG. Preventing HIV among adolescents with oral prep: observations and challenges in the United States and South Africa. J Int AIDS Soc. (2016) 7:21107. doi: 10.7448/IAS.19.7.21107

4. Marrazzo JM, Ramjee G, Richardson BA, Gomez K, Mgodi N, Nair G, et al. Tenofovir-based preexposure prophylaxis For HIV infection among African women. N Engl J Med. (2015) 372:509-18. doi: 10.1056/NEJMoa1402269

5. Global Guidance on Criteria and Processes for Validation: Elimination of Mother-to-Child Transmission of HIV and Syphilis. 2nd edn. Geneva: World Health Organization (2017).

6. Centers for Disease Control and Prevention. Reported STDs in the United States. Atlanta, GA: Centers for Disease Control and Prevention (2019).

7. World Health Organization. Sexually Transmitted Infections (STIs). Available online at: https://www.who.int/news-room/fact-sheets/detail/sexuallytransmitted-infections-(stis) (accessed June 14, 2019).

8. Sully EA, Biddlecom A, Darroch J, Riley T, Ashford LS, Lince-Deroch N, et al. Adding It Up: Investing in Sexual and Reproductive Health 2019. New York, NY: Guttmacher Institute (2020).

9. Boonstra H. Multipurpose Prevention Technologies for the Developing World: U.S. Investment Is Critical. Washington, DC: Guttmacher Institute (2015).

10. Anderson DJ. Population and the environment-time for another contraception revolution. N Engl J Med. (2019) 381:3979. doi: 10.1056/NEJMp1906733

11. Karim S, Baxter C, Frohlich J, Karim QA. The need for multipurpose prevention technologies in sub-saharan Africa. BJOG. (2014) 121:2734. doi: 10.1111/1471-0528.12842

12. Microbicide Trials Network (MTN). Deliver: A study of PrEP and the Dapivirine Ring in Pregnant Women. MTN-042. Microbicide Trials Network. Available online at: mtnstopshiv.org (accessed July 22, 2021).

13. United Nations. Department of Economic and Social Affairs, Population Division. Trends in Contraceptive Use Worldwide (2015). New York, NY: United Nations (2015).

14. Statista Research Department. Global Condom Market Value 2017-2023. Available online at: https:/www.statista.com/statistics/739346/condommarket-value-worldwide/ (accessed November 26, 2020).

15. The Initiative for MPTs. MPT Product Database. Available online at: www.mpts101.org (accessed July 2021).

16. Ipsos. Assessing the Potential of MPTs in South Africa, Uganda and Nigeria: Summary Booklet. Available online at: (accessed July 14, 2021). http://www.theimpt.org/documents/BMGF-AssessingMPTSolutionsBooklet.pdf

17. Van der Straten A, Agot K, Ahmed K, Weinrib R, Browne EN, Manenzhe K, et al. The tablets ring injecions as options (TRIO) study: what young african women choose for future HIV and pregnancy prevention. J Intl AIDS Soc. (2018) 21:e25094. doi: 10.1002/jia2.25094

18. Tolley EE, Li S, ZangenehSZ, Atujuna M, Musara P, Justman J, et al. Acceptability of long-acting HIV prevention product among US and African women: findings from a phase 2 clinical trial HPTN076. J Intl AIDS. (2019) 22:e25048. doi: 10.1002/jia2.25408
Katherine Yang (University of California San Francisco), Sharon Achilles (University of Pittsburgh), and Susanna Moore (IMPT| CAMI Health| Public Health Institute) for their valuable feedback on the ideas outlined in this commentary.

19. Haynes J, Sales J, Sheth A, Lathrop E, Haddad L. Interest in multipurpose prevention technologies to prevention HIV/STIs and unintended pregnancy among young women in the United States. Contraception. (2018) 97:277284. doi: 10.1016/j.contraception.2017.10.006

20. Young Holt B, Morwitz V, Ngo L, Harrison P, Whaley K, Pettifor A, et al. Microbicide preference among young women in California. Jo Women's Health. (2006) 15:281-94. doi: 10.1089/jwh.2006.15.281

21. Ross J, Stover J. Use of modern contraception increases when more methods become available: analysis of evidence from 1982-2009. Glob Health Sci Pract. (2013) 1:203-12. doi: 10.9745/GHSP-D-13-00010

22. U.S. Department of Health and Human Services. Sexually Transmitted Infections National Strategic Plan for the United States: 2021-2025. Washington, DC: Department of Health and Human Services (2020).

23. Britton L, Alspaugh A, Greene M, McLemore M. An evidencebased update on contraception. Am J Nurs. (2020) 20:2233. doi: 10.1097/01.NAJ.0000654304.29632.a7

24. Callahan R, Mehta N, Nanda K, Kopf G. The new contraceptive revolution: developing innovative products outside of industry. Biol Reprod. (2020) 103:157-66. doi: 10.1093/biolre/ioaa067

25. Johnston D, Goldberg E. Preclinical contraceptive development for men and women. Biol Reprod. (2020) 103:147-56. doi: 10.1093/biolre/ioaa076

26. Long J, Lee M, Blithe D. Male contraceptive development: update on novel hormonal and nonhormonal methods. Clin Chem. (2019) 65:15360. doi: 10.1373/clinchem.2018.295089

27. Baeten JM, Palanee-Phillips T, Brown, Schwartz K, Soto-Torres LE, Govender $\mathrm{V}$, et al. Use of a vaginal ring containing dapivirine for HIV-1 prevention in women. N Engl J Med. (2016) 375:2121-32. doi: 10.1056/NEJMoa1506110

28. Nel A, van Niekerk N, Kapiga S, Bekker L-G, Gama C, Gill K, et al. Safety and efficacy of a dapivirine vaginal ring for HIV prevention in women. $N$ Engl J Med. (2016) 375:2133-43. doi: 10.1056/NEJMoa1602046

29. Nel A, van Niekerk N, Van Baelen B, Malherbe M, Mans W, Carter A, et al. Safety, adherence, and HIV-1 seroconversion among women using the dapivirine vaginal ring (DREAM): an open-label, extension study. Lancet HIV. (2021) 8:e77-86. doi: 10.1016/S2352-3018(20)30300-3

30. Baeten JM, Palanee-Phillips T, Mgodi NM, Mayo AJ et al. Safety, uptake, and use of a dapivirine vaginal ring for HIV-1 prevention in African women (HOPE): an open-label, extension study. Lancet HIV. (2021) 8:e87e95. doi: 10.1016/S2352-3018(20)30304-0

31. Landovitz RJ, Donnell D, Clement M, Hanscom B, Cottle L, Coelho L, et al. HPTN 083 FINAL RESULTS: pre-exposure prophylaxis containing longacting injectable cabotegravir is safe and highly effective for cisgender men and transgender women who have sex with men. Available online at: https:// www.hptn.org/sites/default/files/inline-files/HPTN083_PrimaryAIDS2020_ Landovitz-Final_web.pdf (accessed July 22, 2021).

32. HPTN: HIV Prevention Trials Network (HPTN) Announces Initiation of HPTN 084: First Large-Scale Study in Women of a Long-Acting Injectable to Prevent HIV. Available online at: HIV Prevention Trials Network (HPTN) Announces Initiation of HPTN 084: First Large-Scale Study in Women of a Long-Acting Injectable to Prevent HIV The HIV Prevention Trials Network (accessed April 17, 2021).

33. Grant, B, Lama L, Anderson PL, McMahan V, Liu AY, Vargas L, et al. Preexposure chemoprophylaxis for HIV prevention in men who have sex with men. N Engl J Med. (2010) 363:2587-99. doi: 10.1056/NEJMoa1011205

34. Baeten JM, Donnell D, Ndase P, Mugo NR, Campbell JD, Wangisi J, et al. Antiretrovirus prophylaxis for HIV prevention in heterosexual men and women. N Engl J Med. (2012) 367:399-410. doi: 10.1056/NEJMoa1108524

35. Descovy PrEP: Available online at: https://www.fda.gov/news-events/pressannouncements/fda-approves- second-drug-prevent-hiv-infection-partongoing-efforts-end-hiv-epidemic (accessed July 14, 2021). 
36. Buder S, Schöfer H, Meyer T, Bremer V, Kohl PK, Skaletz-Rorowski A, et al. Bacterial sexually transmitted infections. J Dtsch Dermatol Ges. (2019) 287-315. doi: 10.1111/ddg.13804

37. Krupp K, Madhivanan P. Antibiotic resistance in prevalent bacterial and protozoan sexually transmitted infections. Indian J Sex Transm Dis AIDS. (2015) 36:3-8. doi: 10.4103/0253-7184.156680

38. Gulati S, Schoenhofen IC, Lindhout-Djukic T, Schur MJ, Landig CS, Saha S, et al. Therapeutic CMP-nonulosonates against multidrug-resistant neisseria gonorrhoeae. J Immunol. (2020) 204:3283-95. doi: 10.4049/jimmunol.1901398

39. Edwards VL, Smith SB, McComb EJ, Tamarelle J, Ma B, Humphrys MS, et al. The cervicovaginal microbiota-host interaction modulates chlamydia trachomatis infection. mBio. (2019) 10:e0154819. doi: 10.1128/mBio.01548-19

40. Luo F, Shu M, Gong S, Wen Y, He B, Su S, et al. Antiapoptotic activity of Chlamydia trachomatis Pgp3 protein involves activation of the ERK1/2 pathway mediated by upregulation of DJ-1 protein. Pathog Dis. (2019) 77:ftaa003. doi: 10.1093/femspd/ftaa003

41. Rodriguez-Izquierdo I, Gasco S, Muñoz-Fernández MA. High preventive effect of G2-S16 anionic carbosilane dendrimer against sexually transmitted HSV-2 infection. Molecules. (2020) 25:2965. doi: 10.3390/molecules25132965

42. Barrett SE, Teller RS, Forster SP, et al. Extended-duration MK-8591eluting implant as a candidate for HIV treatment and prevention. Antimicrob Agents Chemother. (2018) 62:e01058-18. doi: 10.1128/AAC.0 1058-18

43. Landovitz RJ, Donnell D, Clement M, Hanscom B, Cottle L, Coelho L, et al. HPTN 084 study demonstrates superiority of CAB LA to Oral FTC/TDF for the prevention of HIV. The HIV prevention trials network. Available online at: HIV-Highlights-Press-Conference-Abstracts.pdf(aids2020.org)
44. Sugarman J. Ethical considerations regarding oral PrEP in HIV prevention trials. Curr Opin HIV AIDS. (2016) 11:10915. doi: 10.1097/COH.0000000000000214

45. Young Holt B, Kiarie J, Kopf G, Nanda K, Hemmerling A, Achilles S. Bridging the gap: advancing multipurpose prevention technologies from the lab into the hands of women. Biol Reprod. (2020) 103:286-8. doi: 10.1093/biolre/ioaa085

Author Disclaimer: The contents of this manuscript are the sole responsibility of the authors and do not necessarily reflect the views of USAID, NICHD or the United States Government.

Conflict of Interest: BYH was employed by the Public Health Institute. JT was employed by the company Howpin Consulting. JR was employed by the company NWJ Group, LLC.

Publisher's Note: All claims expressed in this article are solely those of the authors and do not necessarily represent those of their affiliated organizations, or those of the publisher, the editors and the reviewers. Any product that may be evaluated in this article, or claim that may be made by its manufacturer, is not guaranteed or endorsed by the publisher.

Copyright (c) 2021 Young Holt, Turpin and Romano. This is an open-access article distributed under the terms of the Creative Commons Attribution License (CC BY). The use, distribution or reproduction in other forums is permitted, provided the original author(s) and the copyright owner(s) are credited and that the original publication in this journal is cited, in accordance with accepted academic practice. No use, distribution or reproduction is permitted which does not comply with these terms. 